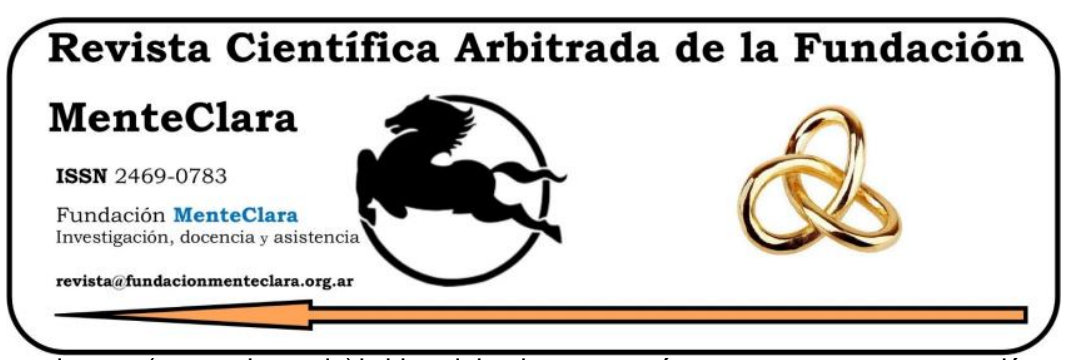

Artículos atravesados por (o cuestionando) la idea del sujeto -y su género- como una construcción psicobiológica de la cultura. Articles driven by (or questioning) the idea of the subject -and their gender- as a cultural psychobiological construction.

Vol. 6 (2021), enero-diciembre ISSN 2469-0783

https: / / datahub.io/ dataset/2021-6-e257

\title{
EL IMPACTO DE LA SEPARACIÓN PARENTAL EN NIÑOS: PERSPECTIVAS TEÓRICAS DE LA PSICOLOGÍA
}

\author{
THE IMPACT OF PARENTAL SEPARATION ON CHILDREN: THEORETICAL \\ PERSPECTIVES IN PSYCHOLOGY
}

Dilcio Dantas Guedes dilcio@gmail.com

Universidad del Salvador, Argentina.

Cómo citar este artículo / Citation: Guedes, D.D. (2021). El impacto de la se paración parental en niños: Perspectivas teóricas de la psicología. Revista Científica Arbitrada de la Fundación MenteClara, Vol. 6 (257). DOI: https://doi.org/10.32351/rca.v6.257

Copyright: (C) 2021 RCAFMC. Este artículo de acceso abierto es distribuido bajo los términos de la licencia Creative Commons Attribution 4.0 International License (CC BY 4.0). Recibido: 06/12/2021. Aceptado: 16/12/2021 Publicación online: 20/12/2021

Conflicto de intereses: Ninguno que declarar.

\section{Resumen}

Revisión de la literatura describe características del impacto de la separación en estudios publicados bajo revisión de pares hechos en diferentes países entre 1955 y 2020. Las diferentes perspectivas teóricas en psicología convergieran que la separación parental se asociaba a reacciones deficitarias o inadaptadas en los niños, que incluian fantasías de abandono y de desvalorización de las figuras parentales identificadas como núcleo de los conflictos.

\section{Abstract}

This literature review describes characteristics of the impact of parental separation in published peer-reviewed studies conducted in diffe rent countries between 1955 and 2020. The different theoretical perspectives in Psychology converged that parental separation was associated with deficit or maladaptive reactions in children, which included fantasies of abandonment and devaluation of the parental figures identified as the core of the conflicts.

Palabras Claves: separación parental; impacto; niños

Keyw ords: parental se paration, impact; children 


\section{Introducción}

Esta revisión fue motivada por el postulado que la situación de separación parental puede configurar un entorno potencialmente disruptivo en el funcionamiento psíquico de niños. La comprensión del impacto de la separación parental puede ser útil en procesos evaluativos a nivel preventivo y de progreso terapéutico. Desde ahí se pregunta: "que consecuencias de la separación parental en niños han sido verificadas en estudios en diferentes perspectivas teóricas de la Psicología”.

\section{Metodologia}

Esta revisión de literatura parte de las investigaciones inquiridas en diferentes plataformas, según los criterios, a saber: (i) publicación sometida a revisión de pares; (ii) integrar los conceptos de "separación parental", “impacto", y "niños". La búsqueda envolvió los trabajos publicados en idiomas castellano, portugués, inglés, francés e italiano. Debido a la escasez de datos, se eligió un encuadre geográfico abierto muestras de múltiples países- publicados desde 1955. Los principales criterios de exclusión fueran los estudios clínicos -la recolección de datos hechos en el ámbito de la clínica-.

\section{Revisión de la Literatura}

\section{1 - Estudios sobre las consecuencias de la separación parental}

Estudios indicaron que el impacto de la separación parental se refleja en la experiencia de los niños como problemas académicos, de conducta, sentimientos de culpa, el desarrollo de los deseos de reconciliación parental, auto imagen y auto estima negativa (Wallerstein, 1975 y 1976; Raschke y Raschke, 1979; Emery, 1982; Wallerstein y Kelly, 1985). 
Además, indicaron que la hostilidad continua entre las figuras parentales, independiente de la modalidad de custodia generaba un entorno marcado por disminución significativa de la disponibilidad emocional que mediatizaba negativamente la resiliencia y el afrontamiento de los niños (Ahroma y Rogers, 1987; Nelson, 1989; Johnston, Kline y Tschann, 1989; Camara y Resnick, 1989; Bender, 1994; Whiteside, 1996, 1998; Jekielek, 1998; Mathis, 1998; Morrison y Coiro, 1999; Madden-Derdich, Leonard y Christopher, 1999; Whiteside y Becker; 2000).

\section{2 - Estudios acerca de la separación parental en la perspectiva teórica sistémica:}

La perspectiva sistémica describe fenómenos específicos en entornos familiares que se enfrentan a una separación conflictiva: los chivos expiatorios, la alineación y el enmarañamiento. El primer refiere al intento de distraer los conflictos interparentales centrándose en los síntomas de los niños; el segundo, el alineamiento, puede conducir a una dinámica de preferencia y rechazo de uno u otra figura parental; el tercer remitiría a la indiferenciación de sentimientos y pensamientos entre los miembros de la familia. Los estudios apuntan que la modalidad de custodia influencia estos sistemas en términos de mayores o menores potenciales de ajustes físicos y psicológicos de los niños a largo plazo (Parish y Taylor, 1979, Parish y Dostal, 1980; Hetherington et al., 1979; Lampel, 1996). 


\section{3 - Estudios acerca de la separación parental en la perspectiva teórica aprendizaje social:}

La perspectiva del aprendizaje social sugiere que los padres que modelan métodos para tratar los desacuerdos y por eso los niños aprenden a manejar sus conflictos entre pares y en relación a sus figuras parentales emulando los comportamientos de sus padres, que estudios evidencian al verificar tendencias de comportamientos conflictivos y actuación agresiva de los niños (Camara y Resnick, 1988; Kline y Johnston, 1991; Amato, 1993; Maccoby et al, 1993; Lampel, 1996; Simons et al., 1999).

\section{4 - Estudios acerca de la separación parental en la perspectiva} cognitiva conductual:

La perspectiva cognitiva conductual sugiere que los niños de familias separadas marcadas por un alto grado de conflicto presentaban medidas de disfunción de conductas más elevadas comparado a las medidas de familias no separadas. La adaptación emocional de los niños se correlacionaba negativamente con el desajuste interparental previo a la separación y positivamente con el ajuste a las funciones parentales posterior a la misma (Kurdek y Berg, 1983. Tschann, Johnston, Kline y Wallerstein, 1989; Cherlin et al, 1991). Loes investigadores y teóricos conductistas postulan que son las percepciones cognitivas de los niños sobre el conflicto interparental que median sus comportamientos y ajuste emocional, donde los esquemas de comportamiento de padres reflejarian en la activación psicofisiológica, asociadas por entornos percibidos con inseguros (Grych y Fincham, 1990; Caron, 2004; Lamela, Figueiredo y Bastos, 2008) 


\section{5 - Estudios acerca de la separación parental en la perspectiva psiconeuroimunoendocrinologico:}

Estudios en el campo psiconeuroimunoendocrinologico acerca del impacto de la separación de figuras de apego con muestras animales reflejan datos encontrados en estudios con humanos: se ha observado que tales impactos se traducen en efectos orgánicos de la adversidad elevación significativa en la actividad adrenocortical, modificación de las sinapsis de dopamina y glutamato en la corteza prefrontal medial, áreas asociativas superiores del prosencéfalo, sinapsis excitadoras e inhibidoras en la corteza cingulada anterior que afectan procesos de toma de decisiones, memoria, respuestas al estrés y control de los impulsos (Hoffman et al., 1995; Ovtscharoff et al., 2006; Mueller y Tronick, 2019;

. Estudios con niños de revelaran mayor incidencia de problemas en el nivel de bienestar biológico que también se asocian con enfermedades en la edad adulta y la senescencia. Tales datos sugieren que el ajuste general de los niños a la separación de los padres sufre un impacto significativo en nivel mental y somático y puede extenderse a otras fases del desarrollo (Maier et al., 2000; Johnson et al., 2002; Cohen et al., 2002; RingsbackWeitoft et al., 2003; Helmreich et al., 2005; Yannakoulia et al., 2008; Roustit et al., 2011; Bambico et al., 2013; Martinón et al., 2017; Olofsson, 2019; Boccia, Cook, Marson y Perdersen, 2021).

\section{6 - Estudios acerca de la separación parental en la perspectiva psicoanalitica:}

Estudios y producciones teóricas en el campo psicoanalítico sugirieran que más que la separación parental, la situación emocional en la familia era el factor determinante en la adaptación del niño. Identificaron que los padres pasan a tener dificultades para contener la lesión de sus 
narcisismos, dañados por la separación, haciendo que los conflictos yóicos en el niño sigan incontenibles (Mahler y Rabinovitch, 1956; Nye, 1957). Así, los objetos parentales ejercen una función interna y externa vital para el niño de forma que la separacion parental interferiría en los procesos internos de los niños (Wylie y Delgado, 1959). En tales entornos, el yo inmaduro de los niños no daría cuenta de resolver los conflictos intrapsíquicos lo que detenían el desarrollo normal, haciéndoles fijados en fantasias pre-edípicas que se demostraban a través de culpa asociada a la percepción de la separación como resultado de los impulsos orales o anales y fantasías omnipotentes. También en ese sentido, la separación parental desequilibraría a función para excitatoria de forma que los impulsos del niño se sobreexcitan y generando angustia (LassondeFontaine, L., 1973, Melanson, 1973). La falla para-excitatoria formaria síntomas y sustentaría la recurrencia de la fantasía de reconciliación de los padres, cómo una forma de revivir el entorno parental triangular originario (Burgner y Gavshon, 1981).

En la perspectiva psicodinámica, los estudios dedicados al fenómeno de la separación familiar a través del análisis de dibujos de niños producidos a partir de consignas de test proyectivos (Dibujo de la Familia - DF; el Dibujo de la Familia Kinetica - DFK; Dibujo de la Familia Kinetica Animal y Dibujo de la Persona Bajo la Lluvia) y dibujos libres indicaran que el impacto de la separación parental en la representación que los niños producían de sí mismos y de sus padres, centrándose en el tamaño y la omisión de las figuras como pautas gráficas (Kelly y Berg, 1978). Tales pautas se configuraban de manera variable según la figura parental ausente (sobre todo del padre), tipo de custodia, genero, edad y auto imagen del niño (Leite-Anderson, 1992). Los estudios también buscaran identificar lo que el Dibujo de la Familia (DF) y el Dibujo de la Familia Kinetica (DFK) evidenciaban: verificaron que mientras el DF 
remetía a una representación de familia idealizada, el DFK remetía a una representación más cerca de imágenes tempranas y nucleares para los niños. Tales pautas parecerán convergir en señales de inhibición, ansiedad, baja auto estima y potencial de vulnerabilidad psicológica entre esos niños (Orgilés, M. y Piñero, J., 2007; Ebling, Pruett y Pruett, 2009; Fan; 2012; Şeno, 2014; Lima, 2014; Atili et al., 2016; Mirivahid et al., 2016; González, 2016; Giotsa y Mitrogiorgou, 2016; Adámy et al., 2018; Mento et al., 2019; Procaccia et al., 2020).

\section{Discusiones de Resultados}

Los estudios de diferentes perspectivas en psicologia sugieren que el impacto de la separación parental se refleja en la experiencia de los niños a nivel académicos, conductual y emocional y puede relacionarse a la modalidad de custodia. Tal relación entre el impacto de la separacion y la modalidad de custodia la adaptación emocionalde los niños, pudiendo, incluso, influir a nivel somático en diferentes fases del desarrollo.

De un punto de vista de estudios psicodinámicos, a través de uso de métodos proyectivos, se indicó que en la mayoría de los estudios, los niños de familias separadas tienden a presentar mayor probabilidad de excluir a los miembros de la familia (sobre todo la figura del padre) y representar experiencias disruptivas de sus entornos. Entre tales representaciones, se evidencian fantasias sobre la reunión de los padres, conflictos de lealtad e inseguridad ilustrados por pautas recurrentes como alejamiento entre las figuras, obstáculos, borrados, rasgos agresivos, de tristeza, y representación corporal incompleta. 


\section{Conclusiones}

Los estudios describieron asociaciones entre las características de continuidad conflictiva interparental en el entorno familiar y la experiencia de sus niños. Al introducir la metodología proyectiva, la mayoría de los autores sugirieran que las respuestas comportamentales y emocionales de los niños eran deficitarias o inadaptadas y se asociaban a las representaciones de fantasías de alejamiento del entorno, desvalorización de las figuras parentales identificadas como núcleo de los conflictos y de sí mismos.

Los estudios reforzaran la idea que el estudio del fenómeno de la separación de los padres se presenta como un proceso multifactorial e idiosincrático y por lo tanto debe tener en cuenta el carácter procesual de la separación (no como un evento) y efectuar triangulación metodológica para abarcar la complejidad del fenómeno. 


\section{Referencias}

Adámy, L., Soltész, K., Szládovics, L., Takács, S., Törő, K.T. (2018). The effects of contested divorce on children's emotional state based on their family drawings and life quality. Integrative Neuroscience Research, 2(1), 1-7.

Attili, G., Toni, A., Di Gianfelice, S., y Roazzi, A. (2011). Children's Family Drawings and Attachment: A Multidimensional Scaling Approach to study Internal Working Models. In: Yael Fisher, y Isaac A. Friedman (Eds.), New Horizons for Facet Theory: Searching for Structure in Content, Spaces and Measurement (pp. 111-122). FTA.

Bambico, F.R., Lacoste, B., Hattan. P.R., y Gobbi, G. (2013) Father absence in the monogamous California mouse impairs social behavior and modifies dopamine and glutamate synapses in the medial prefrontal cortex. Cerebral Cortex, 25 (5), 1163-1175 DOI: $10.1093 /$ cercor/bht310.

Berg, B., y Kelly, R. (1979) The Measured Self-Esteem of Children from Broken, Rejected, and Accepted Families. Journal of Divorce, 2(4), 363-369, DOI: 10.1300/J279v02n04_03

Boccia, M. L., Cook, C., Marson, L., y Pedersen, C. (2021). Parental divorce in childhood is related to lower urinary oxytocin concentrations in adulthood. Journal of Comparative Psychology, 135(1), 74-81. https:// doi.org/10.1037/com0000248

Burgner, M., y Gavshon, A. (1981). Study Group on One-Parent Families. Bul. Anna Freud Centre, 4(4), 292-294

Camara, K. y Resnick, G. (1988). Interparental Conflict and Cooperation: Factors Moderating Children's Post-Divorce Adjustment. ln E. Hetherington y J. Arasteh (Eds.), Impact of divorce, single parenting, and stepparenting on children. (pp.169-195). Lawrence Erlbaum.

Camara, K., y Resnick, G. (1989). Styles of Conflict Re solution and Cooperation between Divorced Parents: Effects on Child Behavior and Adjustment, American Journal of Orthopsychiatry, $59(4), 556-575$.

Caron, C. (2004). Séparation et divorce fortement conflictuels et syndrome d'aliénation parentale : lecture théorique et intervention. Mémoire de Maitrise en Service Social. Université de Montréal.

Cohen, A.J., Adler, N., Kaplan, S.J., Pelcovitz, D., y Mande1, F.S. (2002) Interactional effects of marital status and physical abuse on adole scent psychopathology. Child Abuse y Neglect, 26(3), 277-288. DOI: $10.1016 / \mathrm{s} 0145-2134(01) 00325-8$

Ebling, R., Pruett, K.D., y Pruett, M.K. (2009). "Get over it": perspectives on divorce from young children. Family Court Review, 47(4), 665-681.

Emery, R. (1982). Interparental Conflict and the Children of Discord and Divorce, Psychological Bulletin, 92(2), 310-330.

Fan, R. J. (2012). A study on the kinetic family drawings by children with different family structures. The International Journal of Arts Education, 173-204.

Giotsa, A. y Mitrogiorgou, E. (2016). Representations of Families through the Children's Drawings in Parental Divorce Incidents in Greece. Journal of Child Development Disorder, 2(4). DOI: $10.4172 / 2472-1786.100037$

González, Y. E. C. (2016). Estrategias que minimicen el impacto psicosocial en los niños de madres solteras de 6 a 12 años, del centro de alcance por mi barrio, de Santa Catarina Pínula. Informe de Investigación. Universidad de San Carlos de Guatemala. 
Grych, J. y Fincham, F. (1990). Marital conflict and children's adjustment: A cognitive contextual framework. Psychological Bulletin, 108, $267-290$.

Helmreich, D.L., Parfitt, D.B., Lu, X-Y, Akil, H., y Watson, S.J. (2005) Relation between the hypothalamic-pituitary-thyroid (HPT) axis and the hypothalamic-pituitary-adrenal (HPA) axis during repeated stress. Neuroendocrinology, 81(3), 183-192. DOI: 10.1159/000087001

Hetherington, M.E., Cox, M., y Cox, R. (1979). Play and social interaction in children following divorce. Journal of Social Issues, 35, 79-96.

Hoffman, K.A., Mendoza, S.P., Hennessy, M.B., y Manson, W.A. (1995) Responses of infant Titi monkeys, Callicebus Moloch, to removal of one or both parents: Evidence for paternal attachment. Developmental Psychobiology, 28(7), 399-407. DOI: 10.1002/dev.420280705

Ivanoff, E. (1909) Expériences sur le dessin des écoliers suisses : la corrélation entre l'aptitude au dessin et d'autres capacités. Archives de Psychologie, 8, 97-156.

Jekielek, S. M. (1998). Parental Conflict, Marital Disruption and Children's Emotional Well-Being. Social Forces, 76(3),905-935.

Johnson, J.G., Cohen, P., Kasen, S, y Brook, J.S. (2002) Childhood adversities associated with risk for eating disorders or weight problems during adolescence or early adulthood. The American Journal of Psychiatry, 159(3), 394-400. DOI: 10.1176/appi .ajp.159.3.394

Johnston, J., Kline, M., y Tschann, J. (1989). Ongoing Post-Divorce Conflict: Effects on Children of Joint Custody and Frequent Access. American Journal of Orthopsychiatry, 59(4), 576-592.

Kacenelenbogen, N., Dramaix-Wilmet, M., Schetgen, M., Roland, M., y Godin, I. (2016). Parental se paration: a risk for the psychomotor development of children aged 28 to 32 months? A cross-sectional study. BMC pediatrics, 16, 89. DOI: 10.1186/s12887-016-0621-y

Kelly, J. B., y Wallerstein, J. S. (1976). The effects of parental divorce: Experiences of the child in early latency. American Journal of Orthopsychiatry, 46(1), 20-32. https:/ / doi.org/10.1111/j.1939-0025.1976.tb01224.x

Kelly, R., y Berg, B. (1978). Measuring children's reactions to divorce. Journal of Clinical Psychology, 34(1), 215-221.

Kline, M., y Johnston, J.R. (1991). The long shadow of marital conflict: A model of children's postdivorce adjustment. Journal of Marriage and the Family, 53,297-309

Lamela, D.; Figueiredo, D.; y Bastos, A. (2010). Adaptação ao divórcio e relações coparentais: contributos da teoria da vinculação. Psicologia Reflexão e Critica, 23(3), 562-574.

Lampe1, A. (1996). Children's alignment with parents in highly conflicted custody case s. Family and Conciliation Courts Review, 34(2),229-239.

Lassonde-Fontaine, L. (1973). Les effets de l'absence du père chez le garçon au cours de sa période œdipienne (étude exploratoire). Mémoire de Maîtrise non publié, Université de Montréal.

Leite-Anderson, D.A. (1992). Effects of divorce on children's family drawings by children in two age groups. Masters of Sciences Dissertation. Emporia State University.

Lima, B.E.M. (2014). A Dupla Lua: indicadores de validação junto de filhos de famílias divorcia das. Dissertação de Mestrado Psicologia. Universidade Católica Portuguesa.

Madden-Derdich, D.A., Leonard, S.A., y Christopher, F.S. (1999). Boundary Ambiguity and Coparental Conflict After Divorce: An Empirical Test of the Family Systems Model of the Divorce Process. Journal of Marriage and the Family, 61, 588-98.

Mahler, M. S., y Rabinovitch, R. (1956). The effects of marital conflict on child development. En Eisenstein, V. W. (eds.), Neurotic Interaction in Marriage (pp. 44-56). Basic Books. 
Maier E. H., y Lachman, M.E. (2000) Consequences of early parental loss and se paration for health and well-being in midlife. International Journal of Behavioral Development 24(2), $183-189$. DOI: $10.1080 / 016502500383304$

Martinón, J.M., Fariña, F., Corras, T., Seijo, D., Souto, A., y Novo, M. (2017). Impacto de la ruptura de los progenitores en el estado de salud fisica de los hijos. European Journal of Education and Psychology, 10 (1), 9-14. DOI: 10.1016/j.ejeps.2016.10.002

Mathis, R. (1998). Couples from Hell: Undifferentiated Spouses in Divorce Mediation. Mediation Quarterly, 16, 37-49.

Melanson, D. (1973). Etude du dessin de famille chez des enfants de parents séparés, privés de leur père. Mémoire de Maîtrise non publié, Université de Montréal.

Mento, C., Modicamore, D., La Torre, D., Sivestri, M. C., y Rizzo, A. (2019). Family drawing and psychological vulnerability in children's representations of parental divorce. Cogent Psychology, 6. DOI: 10.1080/23311908.2019.1654723

Mirivahid, S.M., Abbassi, H., y Heydarnia, A. A. (2016). Comparison of kinetic family drawing indicators in intact and divorced children. Journal of Fundamentals of Mental Health, 18(6), 329-37.

Mueller, I., y Tronick, E. (2019). Early Life Exposure to Violence: Developmental Consequences on Brain and Behavior. Frontiers in Behavioral Neuroscience, 13, 156. https:/ / doi.org/ 10.3389/fn beh.2019.00156

Nelson, R. (1989). Parental Hostility, Conflict and Communication in Joint and Sole Custody Families. Journal of Divorce, 13(2), 145-157.

Nye, I. F. (1957). Child adjustment in broken and in unhappy unbroken homes. Marriage and Family Living, 19,356-361.

Olofsson, E. (2019). Children of Divorce: Long-Term Psychological Effects and Neurological Consequences. Bachelor Degree Project in Cognitive Neuroscience, University of Skövde.

Orgilés, M. y Piñero, J. (2007). ¿Cómo perciben los niños la ruptura familiar? Aplicación del test del Dibujo de la Familia en una muestra de hijos de padres separados. Psicopatología y Salud Mental, 9, 49-55.

Ovtscharoff, W., Helmeke, C., y Braun, K. (2006) Lack of paternal care affects synaptic development in the anterior cingulate cortex. Brain Research, 1116(1), 58-63. DOI: $10.1016 /$ j.brainres.2006.07.106

Parish, T., y Dostal, J.W. (1980). Evaluations of self and parent figures by children from intact, divorced, and reconstituted families. Journal of Youth and Adolescence, 9, 347-351.

Parish, T.H., y Taylor, J.C. (1979). The impact of divorce and subsequent father absence on children's and adolescent's self-concept. Journal of Youth and Adole scence, 8, 427-432.

Procaccia, R., Miragoli, S., Camisasca, E.y Di Blasio, P. (2020) Children in Conflictual Separations: Representations of Self and Family through the Blacky Pictures'Test. Journal of Forensic Psychology Research and Practice, 20(2), 185-204, DOI: 10.1080/24732850.2020.1714404

Raschke, H., y Raschke, V. (1979). Family Conflict and Children's Self-Concepts: A Comparison of Intact and Single-Parent Families. Journal of Marriage and the Family, 41, 367-374.

Roustit, C., Campoy, E., Renahy, E., et al. (2011) Family social environment in childhood and selfrated health in young adulthood. BMC Public Health, 11(949). DOI: 10.1186/1471-2458-11949 
Şeno, T. (2014). Research about Family Perceptions via Pictures of Children from Problematic Families or Having Divorced Parents. Procedia. Social and Behavioral Sciences, 116, 2895 2902.

Simons, R., Lin, K, Gordon, L. Conger, R. y Lorenz, F. (1999). Explaining the higher incidence of adjustment problems among children of divorce compared with those in two-parent families. Journal of Marriage y the Family, 61(4), 1020-1034

Spigelman, G., Spigelman, A., y Englesson, L. (1992). Analysis of family drawings: A comparison between children from divorce and non-divorce families. Journal of Divorce and Remarriage, $18,31-54$.

Tschann, J. M., Johnston, J. R., Kline, M., y Wallerstein (1989). Family process and children's functioning after divorce. Journal of Marriage and the Family, 51, 431-444.

Wallerstein, J., y Kelly, J. (1975). The effects of parental divorce: experiences of the preschool child. Journal of the American Academy of Children Psychiatric, 14(4), 600-616.

Wallerstein, J., y Kelly, J. (1976). The effects of parental divorce: Experiences of the child in later latency. American Journal of Orthopsychiatry, 46(1), 20-32

Yannakoulia, M., Papanikolaou, K., Hatzopoulou, I., Efstathiou, E., Papoutsakis, C., y Dedoussis, G.V. (2008) Association between family divorce and children's BMI and meal patterns: The GENDAI study. Obesity, 16(6), 1382-1387. 